\title{
Retraction
}

\section{Retracted: The Stability of Gauss Model Having One-Prey and Two-Predators}

\begin{abstract}
Applied Analysis
Received 27 August 2013; Accepted 27 August 2013

Copyright (C) 2013 Abstract and Applied Analysis. This is an open access article distributed under the Creative Commons Attribution License, which permits unrestricted use, distribution, and reproduction in any medium, provided the original work is properly cited.
\end{abstract}

This article has been retracted as it is essentially identical in title and technical content with a published paper in Journal of Nonlinear Analysis and Optimization published in 2011. The article was submitted on the same day in two different journals; Abstract and Applied Analysis and Journal of Nonlinear Analysis and Optimization, without the knowledge of Dr. Dumitru Baleanu [1].

\section{References}

[1] A. Farajzadeh, M. H. R. Doust, F. Haghighifar, and D. Baleanu, "The stability of Gauss model having one-prey and twopredators," Abstract and Applied Analysis, vol. 2012, Article ID 219640, 9 pages, 2012. 


\title{
Research Article
}

\section{The Stability of Gauss Model Having One-Prey and Two-Predators}

\author{
A. Farajzadeh, ${ }^{1}$ M. H. Rahmani Doust, ${ }^{2}$ F. Haghighifar, ${ }^{2}$ \\ and D. Baleanu ${ }^{3,4}$ \\ ${ }^{1}$ Department of Mathematics, Razi University, Kermanshah 67149, Iran \\ ${ }^{2}$ Department of Mathematics, Ilam University, Ilam 69315-516, Iran \\ ${ }^{3}$ Department of Mathematics and Computer Sciences, Cankaya University, 06530 Ankara, Turkey \\ ${ }^{4}$ Institute of Space Sciences, Bucharest, Magurele, Romania
}

Correspondence should be addressed to D. Baleanu, dumitru@cankaya.edu.tr

Received 22 August 2011; Revised 10 November 2011; Accepted 11 November 2011

Academic Editor: Muhammad Aslam Noor

Copyright (C) 2012 A. Farajzadeh et al. This is an open access article distributed under the Creative Commons Attribution License, which permits unrestricted use, distribution, and reproduction in any medium, provided the original work is properly cited.

The study of the dynamics of predator-prey interactions can be recognized as a major issue in mathematical biology. In the present paper, some Gauss predator-prey models in which three ecologically interacting species have been considered and the behavior of their solutions in the stability aspect have been investigated. The main aim of this paper is to consider the local and global stability properties of the equilibrium points for represented systems. Finally, stability of some examples of Gauss model with one prey and two predators is discussed.

\section{Introduction}

Gauss is one of the well-known scientists who studied in various area in mathematics such as mathematical biology and mathematical ecology. One of his famous models is predatorprey problem in which were obtained the fundamental results in order to be interpreted and analyzed by him. In 1934, Gauss introduced his standard model in mathematical biology. After two years, he and Smaragdov studied a generalization of the following model as a model for predator-prey interactions:

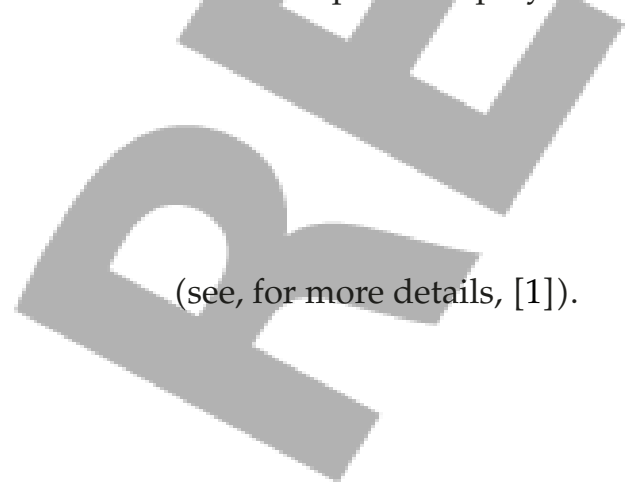

$$
\begin{gathered}
\frac{d x}{d t}=a x-y p(x), \\
\frac{d y}{d t}=y(-\gamma+c p(x))
\end{gathered}
$$


More general form of this model known as an intermediate model of predator-prey interactions is as follows:

$$
\begin{aligned}
& \frac{d x}{d t}=x g(x)-y p(x), \\
& \frac{d y}{d t}=y(-\gamma+q(x)) .
\end{aligned}
$$

Since the predator-prey system is investigated and extended frequently, one of the more investigated extensions of predator-prey system is this model with two preys and one predator. One may see some analysis of predator-prey system having two preys and one predator in references such as [2-8]. Elabbasy and Lisena studied dynamics of periodic predator-prey system having two preys and one predator $[2,6]$. Gakkhar and Singh investigated food web model consisting of two preys and one predator which was a harvested factor [3]. The predator-prey system with two preys and one predator consisting of effort rate harvested factor is studied in $[4,5,7]$. A dynamic behavior of the Holling-II system with two preys and one predator system and with impulsive effect concerning biological control is investigated in [8].

\section{The Predator-Prey Gauss Model with One Prey and Two Predators}

Let us consider a system of two predator species living in an ecosystem independently and each species baits the prey. The Gauss model with one prey and two predators may be written as follows:

$$
\begin{aligned}
& \frac{d x}{d t}=a_{1} x-y p_{1}(x)-z p_{2}(x), \\
& \frac{d y}{d t}=-\gamma_{1} y+c_{1} y p_{1}(x) \\
& \frac{d z}{d t}=-\gamma_{2} z+c_{2} z p_{2}(x)
\end{aligned}
$$

where $x$ is the density of prey species, and $y$ and $z$ are the densities of predators species. In this system, all of coefficients $a_{1}, \gamma_{1}, \gamma_{2}, c_{1}$, and $c_{2}$ are positive and constant. Moreover, the prey enhances in absence of predators species and this increasing is limited by terms $-y p_{1}(x)$ and $-z p_{2}(x)$. In the absence of prey, density of predators populations decrease by exponential growth rate and the prey has positive efficiency on predator population to be a positive sign; the terms $c_{1} y p_{1}(x)$ and $c_{2} z p_{2}(x)$ prove this claim.

For example, consider two species fox and eagle living in an ecosystem and each of the two species baits of rabbit species. In addition to assumptions of Gauss's model, assume that $p_{1}(x)$ and $p_{2}(x)$ have properties of $p(x)$ in the Gauss model (1.1).

In system (2.1), the following properties are held.

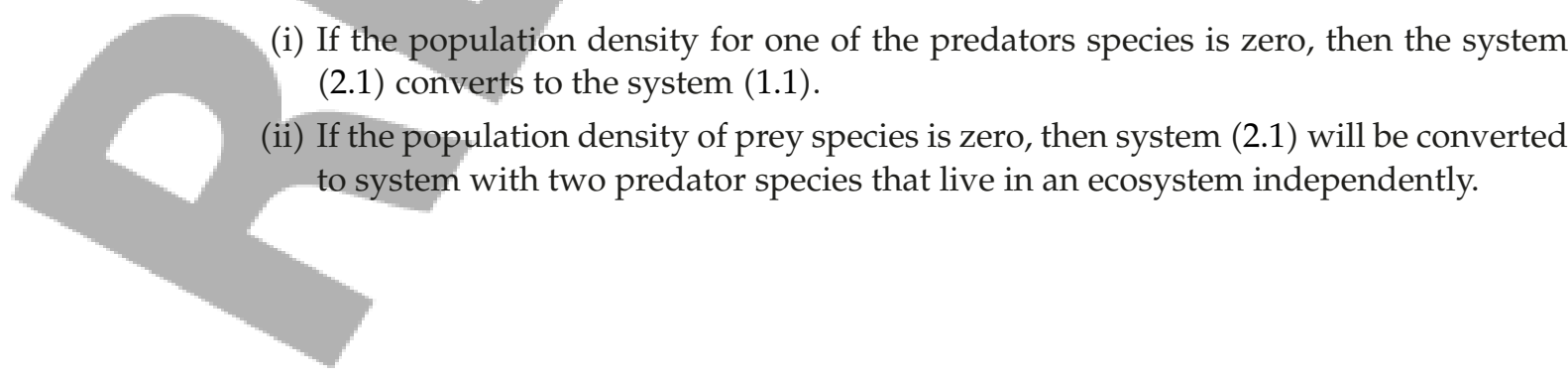


(iii) Let the population density of two species be zero, then system (2.1) will be converted to an equation of growth rate.

(iv) The solutions orbit of system (2.1) is located in the following set:

$$
\operatorname{int} R_{+}^{3}=\operatorname{int}\left\{x_{i} \mid x_{i} \geqslant 0, i=1,2,3\right\} \text {. }
$$

The terms $p_{1}(x)$ and $p_{2}(x)$ have the properties described as follows:

(i) $p_{1}(0)=0, p_{1}(x)$ is continuous and differentiable for $x \geq 0, d p_{1}(x) / d x>0$,

(ii) $p_{2}(0)=0, p_{2}(x)$ is continuous and differentiable for $x \geq 0, d p_{2}(x) / d x>0$.

\section{Local Stability}

We use the linearisation method to study the stability of the system (2.1). By this means, we calculate the Jacobian matrix, which may be found as follows:

$$
\left.J\right|_{(x, y, z)}=\left(\begin{array}{ccc}
a_{1}-y \frac{d p_{1}(x)}{d x}-z \frac{d p_{2}(x)}{d x} & -p_{1}(x) & -p_{2}(x) \\
c_{1} y \frac{d p_{1}(x)}{d x} & -\gamma_{1}+c_{1} p_{1}(x) & 0 \\
c_{2} z \frac{d p_{2}(x)}{d x} & 0 & -\gamma_{2}+c_{2} p_{2}(x)
\end{array}\right) .
$$

Now, let $(\bar{x}, \bar{y}, \bar{z})$ be the equilibrium point of system (2.1). Then

$$
A=\left.J\right|_{(\bar{x}, \bar{y}, \bar{z})}=\left(\begin{array}{ccc}
a_{1}-\bar{y} \frac{d p_{1}(\bar{x})}{d x}-\bar{z} \frac{d p_{2}(\bar{x})}{d x} & -p_{1}(\bar{x}) & -p_{2}(\bar{x}) \\
c_{1} y \frac{d p_{1}(\bar{x})}{d x} & -\gamma_{1}+c_{1} p_{1}(\bar{x}) & 0 \\
c_{2} z \frac{d p_{2}(\bar{x})}{d x} & 0 & -\gamma_{2}+c_{2} p_{2}(\bar{x})
\end{array}\right) .
$$

And so, if $\operatorname{tr} A<0$ and $\operatorname{det} A>0$, therefore, the system (2.1) is locally asymptotically stable at the equilibrium point $(\bar{x}, \bar{y}, \bar{z})$.

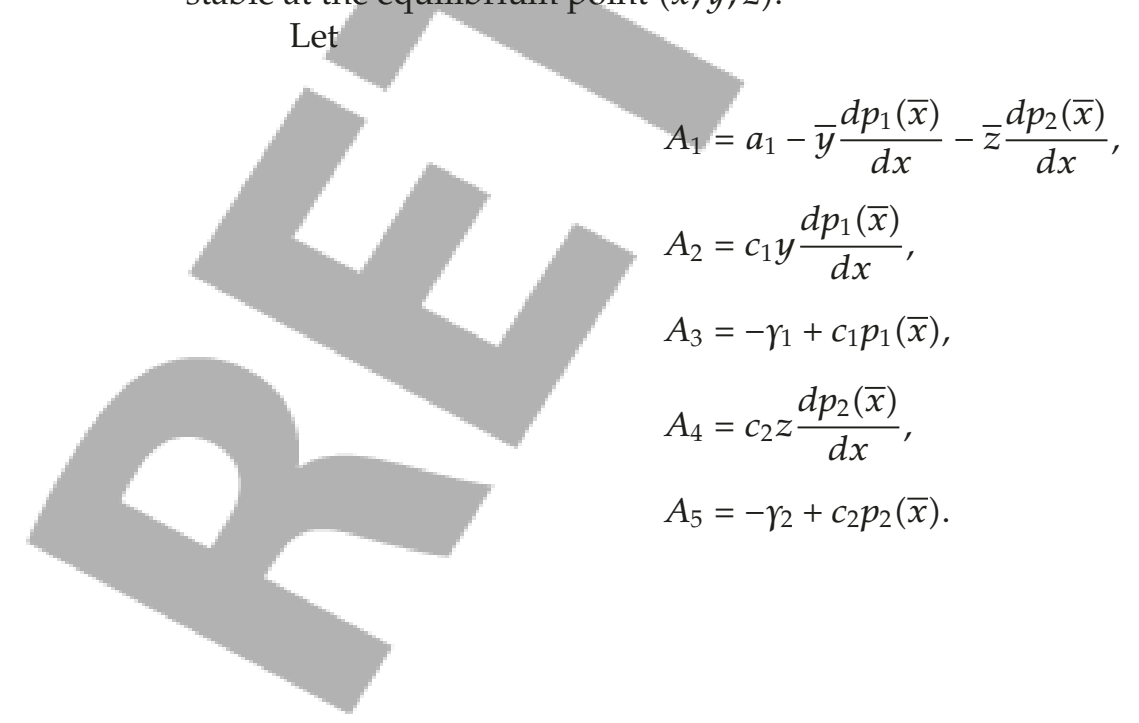


Then,

$$
A=\left.J\right|_{(\bar{x}, \bar{y}, \bar{z})}=\left(\begin{array}{ccc}
A_{1} & -p_{2}(\bar{x}) & -p_{1}(\bar{x}) \\
A_{2} & A_{3} & 0 \\
A_{4} & 0 & A_{5}
\end{array}\right) .
$$

Therefore,

$$
\begin{gathered}
\operatorname{det} A=A_{1} A_{3} A_{5}+p_{2}(\bar{x}) A_{2} A_{5}+p_{1}(\bar{x}) A_{3} A_{4}, \\
\operatorname{tr} A=A_{1}+A_{3}+A_{5} .
\end{gathered}
$$

Hence, the system (2.1) is locally asymptotically stable if $A_{1} A_{3} A_{5}+p_{2}(\bar{x}) A_{2} A_{5}+p_{1}(\bar{x}) A_{3} A_{4}>0$ and $A_{1}+A_{3}+A_{5}<0$.

So, the following proposition is proved.

Proposition 3.1. Let $A_{1} A_{3} A_{5}+p_{2}(\bar{x}) A_{2} A_{5}+p_{1}(\bar{x}) A_{3} A_{4}>0$ and $A_{1}+A_{3}+A_{5}<0$. The system (2.1) is locally asymptotically stable at its equilibrium point $(\bar{x}, \bar{y}, \bar{z})$, whenever it exists.

\section{Global Stability}

In this section, we will prove the global stability of the system (2.1) by constructing a suitable Lyapunov function.

Theorem 4.1. The system (2.1) is globally asymptotically stable at equilibrium point $(\bar{x}, \bar{y}, \bar{z})$, where $x<\bar{x}, y>\bar{y}$ and $z>\bar{z}$.

Proof. Let us consider a suitable Lyapunov function

$$
v(x, y, z)=x+h y+k z
$$

where $h=1 / c_{1}$ and $k=1 / c_{2}$. Obviously $v$ is a positive definite. Now take the derivative from the last Lyapunov system with respect to the time $t$. So we have

$$
\frac{d v}{d t}=\frac{d x}{d t}+h \frac{d y}{d t}+k \frac{d z}{d t}
$$

By substituting $d x / d t, d y / d t$ and $d z / d t$ in the system (2.1), we obtain the said derivative as

\section{follows:}

$$
\frac{d v}{d t}=\left[a_{1} x-y p_{1}(x)-z p_{2}(x)\right]+h\left[-\gamma_{1} y+c_{1} y p_{1}(x)\right]+k\left[-\gamma_{2} z+c_{2} z p_{2}(x)\right]
$$


Since $(\bar{x}, \bar{y}, \bar{z})$ is an equilibrium point for system $(2.1)$, so we can add the zero equations to $d v / d t$ as follows:

$$
\begin{aligned}
\frac{d v}{d t}= & {\left[a_{1} x-y p_{1}(x)-z p_{2}(x)\right]+h\left[-\gamma_{1} y+c_{1} y p_{1}(x)\right]+k\left[-\gamma_{2} z+c_{2} z p_{2}(x)\right] } \\
= & {\left[a_{1} x-y p_{1}(x)-z p_{2}(x)-\left[a_{1} \bar{x}-\bar{y} p_{1}(\bar{x})-\bar{z} p_{2}(\bar{x})\right]\right] } \\
& +h\left[-\gamma_{1} y+c_{1} y p_{1}(x)-\left[-\gamma_{1} \bar{y}+c_{1} \bar{y} p_{1}(\bar{x})\right]\right]+k\left[-\gamma_{2} z+c_{2} z p_{2}(x)-\left[-\gamma_{2} \bar{z}+c_{2} \bar{z} p_{2}(\bar{x})\right]\right] \\
= & {\left[a_{1}(x-\bar{x})-y p_{1}(x)+\bar{y} p_{1}(\bar{x})-z p_{2}(x)+\bar{z} p_{2}(\bar{x})\right] } \\
& +h\left[-\gamma_{1}(y-\bar{y})+c_{1} y p_{1}(x)-c_{1} \bar{y} p_{1}(\bar{x})\right]+k\left[-\gamma_{2}(z-\bar{z})+c_{2} z p_{2}(x)-c_{2} \bar{z} p_{2}(\bar{x})\right],
\end{aligned}
$$

and by putting $h=1 / c_{1}$ and $k=1 / c_{2}$, we find out

$$
\frac{d v}{d t}=a_{1}(x-\bar{x})-\frac{r_{1}}{c_{1}}(y-\bar{y})-\frac{\gamma_{2}}{c_{2}}(z-\bar{z})
$$

Therefore, $d v / d t<0$ if $x<\bar{x}, y>\bar{y}$ and $z>\bar{z}$. This completes the proof.

\section{Analysis of Examples}

In this section, we present some examples of Gauss's model and analyze the stability of them.

\subsection{Analysis of Example 1}

Consider the following system:

In the above system, all coefficients $a_{1}, a_{2}, a_{3}, b_{1}, b_{2}, c_{1}$, and $c_{2}$ are positive constants. In the system (5.1), efficiency of the predator species on the preys species and also efficiency of the preys species on the predator species are linear. The points $(0,0,0),\left(b_{1} / b_{2}, a_{1} / a_{2}, 0\right)$, and $\left(c_{1} / c_{2}, 0, a_{1} / a_{3}\right)$ are equilibrium points of the system (5.1) which may be analyzed by using the Jacobian matrix. We first calculate the Jacobian matrix of system (5.1) as follows:

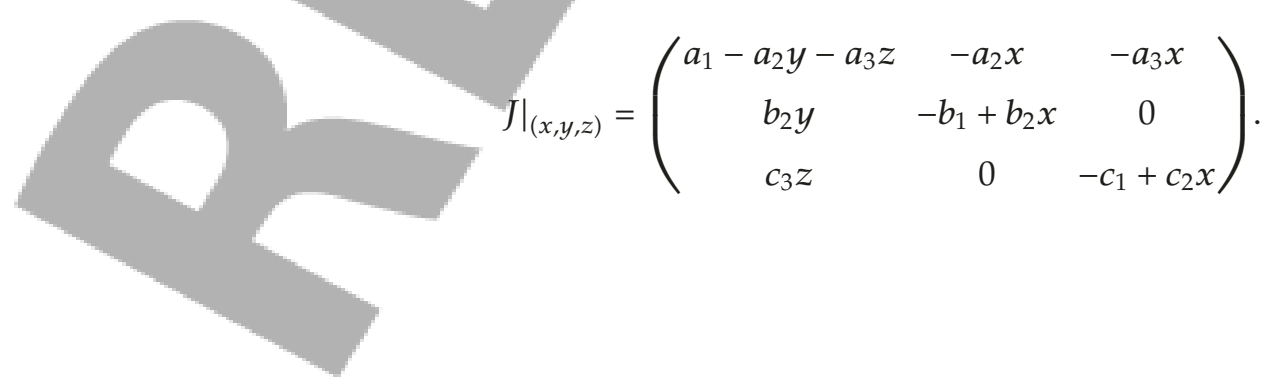


So

$$
\left.J\right|_{(0,0,0)}=\left(\begin{array}{ccc}
a_{1} & 0 & 0 \\
0 & -b_{1} & 0 \\
0 & 0 & -c_{1}
\end{array}\right) .
$$

Thus, the origin is a saddle point for the system (5.1).

The Jacobian matrix for the above system at the equilibrium point $\left(b_{1} / b_{2}, a_{1} / a_{2}, 0\right)$ is given by

$$
A_{1}=\left.J\right|_{\left(b_{1} / b_{2}, a_{1} / a_{2}, 0\right)}=\left(\begin{array}{ccc}
0 & -\frac{a_{2} b_{1}}{b_{2}} & -\frac{a_{3} b_{1}}{b_{2}} \\
\frac{b_{2} a_{1}}{a_{2}} & 0 & 0 \\
0 & 0 & \frac{b_{1} c_{2}}{b_{2}}
\end{array}\right)
$$

Thus,

$$
\operatorname{det}\left(A_{1}-\lambda I\right)=\left(\frac{b_{1} c_{2}}{b_{2}}-\lambda\right)\left(\lambda^{2}+a_{1} b_{1}\right) .
$$

Hence, the equilibrium point $\left(b_{1} / b_{2}, a_{1} / a_{2}, 0\right)$ is hyperbolic point for the system (5.1).

The Jacobian matrix at the equilibrium point $\left(c_{1} / c_{2}, 0, a_{1} / a_{3}\right)$ is obtained by

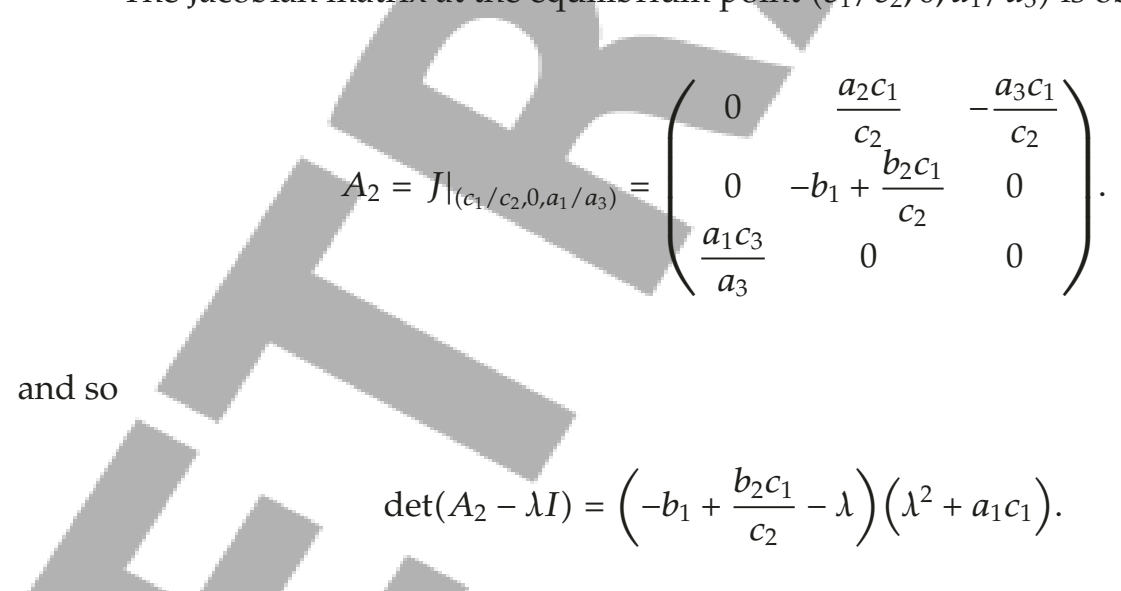

Thus, the equilibrium point $\left(c_{1} / c_{2}, 0, a_{1} / a_{3}\right)$ is hyperbolic point for the system (5.1). In fact, we worked out the following proposition.

Proposition 5.1. The following statements are true for the system (5.1).

(i) The equilibrium point $(0,0,0)$ is a saddle point.

(ii) The equilibrium points $\left(b_{1} / b_{2}, a_{1} / a_{2}, 0\right)$ and $\left(c_{1} / c_{2}, 0, a_{1} / a_{3}\right)$ are hyperbolic points. 
Abstract and Applied Analysis

\subsection{Analysis of Example 2}

In the second example, we consider that there are two predator species which live in an ecosystem independently, but their food is the same, that is, one species prey that has interaction between members species whose mathematical model is as follows:

$$
\begin{aligned}
& \frac{d x}{d t}=x\left(a_{1}-a_{2} x-a_{3} y-a_{4} z\right), \\
& \frac{d y}{d t}=y\left(1-\frac{y}{v_{1} x}\right), \\
& \frac{d z}{d t}=z\left(1-\frac{z}{v_{2} x}\right) .
\end{aligned}
$$

It is clear that $x \neq 0$.

Moreover, the equilibrium points of system (5.8) are given by

$$
\left(\frac{a_{1}}{a_{2}}, 0,0\right), \quad\left(\frac{a_{1}}{a_{2}+a_{4} v_{2}}, 0, \frac{v_{2} a_{1}}{a_{2}+a_{4} v_{2}}\right), \quad\left(\frac{a_{1}}{a_{2}+a_{3} v_{1}}, \frac{a_{1} v_{1}}{a_{2}+a_{3} v_{1}}, 0\right) \text {. }
$$

Also, its Jacobian matrix is as follows:

$$
\left.J\right|_{(x, y, z)}=\left(\begin{array}{ccc}
a_{1}-2 a_{2} x-a_{3} y-a_{4} z & -a_{3} x & -a_{4} x \\
\frac{y^{2}}{v_{1} x^{2}} & 1-\frac{2 y}{v_{1} x} & 0 \\
\frac{z^{2}}{v_{2} x^{2}} & 0 & 1-2 \frac{z}{v_{2} x}
\end{array}\right) .
$$

Now by substituting the equilibrium point $\left(a_{1} / a_{2}, 0,0\right)$ and simplifying, the Jacobian matrix becomes

$$
\left.J\right|_{\left(a_{1} / a_{2}, 0,0\right)}=\left(\begin{array}{ccc}
-a_{1} & -\frac{a_{1} a_{3}}{a_{2}} & -\frac{a_{1} a_{4}}{a_{2}} \\
0 & 1 & 0 \\
0 & 0 & 1
\end{array}\right) .
$$

The eigenvalues of the above matrix are $\lambda_{1}=\lambda_{2}=0$ and $\lambda_{3}=-a_{1}$. Thus, the system (5.8) at equilibrium point $\left(a_{1} / a_{2}, 0,0\right)$ is stable.

Also, the Jacobian matrix at the equilibrium point $\left(a_{1} /\left(a_{2}+a_{4} v_{2}\right), 0, v_{2} a_{1} /\left(a_{2}+a_{4} v_{2}\right)\right)$ is given by

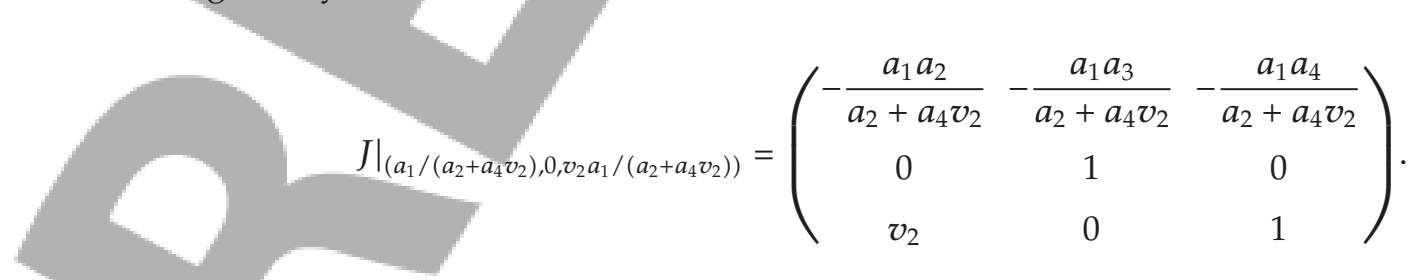


The eigenvalues of the above matrix are $\lambda_{1}=1, \lambda_{2}=-1$ and $\lambda_{3}=a_{1} a_{2} /\left(a_{2}+a_{4} v_{2}\right)$, and so the equilibrium point $\left(a_{1} / a_{2}, 0,0\right)$ is a saddle point for the system (5.8).

Finally, the last equilibrium point is given by

$$
\left(\frac{a_{1}}{a_{2}+a_{3} v_{1}}, \frac{a_{1} v_{1}}{a_{2}+a_{3} v_{1}}, 0\right)
$$

where its Jacobian matrix is as follows:

$$
A=\left.J\right|_{\left(a_{1} /\left(a_{2}+a_{3} v_{1}\right), a_{1} v_{1} /\left(a_{2}+a_{3} v_{1}\right), 0\right)}=\left(\begin{array}{ccc}
-\frac{a_{1} a_{2}}{a_{2}+a_{3} v_{1}} & -\frac{a_{1} a_{3}}{a_{2}+a_{3} v_{1}} & -\frac{a_{1} a_{4}}{a_{2}+a_{3} v_{2}} \\
v_{1} & -1 & 0 \\
0 & 0 & 1
\end{array}\right) .
$$

and so

$$
\begin{gathered}
\operatorname{tr} A=-\frac{a_{1} a_{2}}{a_{2}+a_{3} v_{1}}<0 \\
\operatorname{det} A=\frac{a_{1} a_{2}}{a_{2}+a_{3} v_{1}}+\frac{a_{1} a_{3} v_{1}}{a_{2}+a_{3} v_{1}}>0
\end{gathered}
$$

Therefore, the system (5.8) is locally asymptotically stable at the above equilibrium point.

Therefore, we can summarize the above facts in the following proposition.

Proposition 5.2. For the system (5.8), the following statements are held.

(i) It has three equilibrium points which are as follows:

$$
\left(\frac{a_{1}}{a_{2}}, 0,0\right), \quad\left(\frac{a_{1}}{a_{2}+a_{4} v_{2}}, 0, \frac{v_{2} a_{1}}{a_{2}+a_{4} v_{2}}\right), \quad\left(\frac{a_{1}}{a_{2}+a_{3} v_{1}}, \frac{a_{1} v_{1}}{a_{2}+a_{3} v_{1}}, 0\right) .
$$

(ii) The said system is stable at the equilibrium point $\left(a_{1} / a_{2}, 0,0\right)$.

(iii) The point $\left(a_{1} /\left(a_{2}+a_{4} v_{2}\right), 0, v_{2} a_{1} /\left(a_{2}+a_{4} v_{2}\right)\right)$ is saddle point for the above system.

(iv) The point $\left(a_{1} /\left(a_{2}+a_{3} v_{1}\right), a_{1} v_{1} /\left(a_{2}+a_{3} v_{1}\right), 0\right)$ is locally asymptotically stable for the said system.

\section{Conclusion}

By adding some inequalities, one may make the Gauss system having one prey and two predators asymptotically stable globally. Furthermore, it can be guessed that the generalization of the Gauss system with existing $n$-preys and $m$-predators is globally asymptotically stable provided the following statements hold.

(i) The density of each prey is less than the corresponding component in related equilibrium point. 
Abstract and Applied Analysis

(ii) The density of each predator is greater than the corresponding component in related equilibrium point. Moreover, one may determine the local stability for some particular model of the Gauss system having one prey and two predators such as (5.1) and (5.8) by using the linearisation method.

\section{References}

[1] T. A. Burton, Volterra Integral and Differential Equations, vol. 167, Academic Press, Orlando, Fla, USA, 1983.

[2] E. M. Elabbasy and S. H. Saker, "Dynamics of a class of non-autonomous systems of two noninteracting preys with common predator," Journal of Applied Mathematics E Computing, vol. 17, no. 1-2, pp. 195-215, 2005.

[3] S. Gakkhar and B. Singh, "The dynamics of a food web consisting of two preys and a harvesting predator," Chaos, Solitons and Fractals, vol. 34, no. 4, pp. 1346-1356, 2007.

[4] T. K. Kar and K. S. Chaudhuri, "Harvesting in a two-prey one-predator fishery: a bioeconomic model," The Australian \& New Zealand Industrial and Applied Mathematics Journal, vol. 45, no. 3, pp. 443-456, 2004.

[5] S. Kumar, S. K. Srivastava, and P. Chingakham, "Hopf bifurcation and stability analysis in a harvested one-predator-two-prey model," Applied Mathematics and Computation, vol. 129, no. 1, pp. 107-118, 2002.

[6] B. Lisena, "Asymptotic behaviour in periodic three species predator-prey systems," Annali di Matematica Pura ed Applicata, vol. 186, no. 1, pp. 85-98, 2007.

[7] G. P. Samanta, D. Manna, and A. Maiti, "Bioeconomic modelling of a three-species fishery with switching effect," Journal of Applied Mathematics E Computing, vol. 12, no. 1-2, pp. 219-231, 2003.

[8] X. Song and Y. Li, "Dynamic complexities of a Holling II two-prey one-predator system with impulsive effect," Chaos, Solitons and Fractals, vol. 33, no. 2, pp. 463-478, 2007. 